\title{
X.
}

Mitteilung aus der Klinik ftir Ohren-, Nasen- und Halskrankheiten des Kommunehospitals zu Kopenhagen (Direktor: Prof. Dr. Holger Mygind).

\section{Die durch adenoide Vegetationen hervorgerufenen Symptome, Folgekrankheiten und Komplikationen.} Von Stabsarzt Joh. Fred. Fiseher, Assistent an der Klinik.

Wenn es gilt, die durch adenoide Vegetationen hervorgerufenen Symptome und Komplikationen zu beleuchten und insonderheit die Häufigkeit zu beurteilen, mit welcher dieselben auftreten, so wird zu diesem Zweeke ein Material vortrefflich geeignet sein, welches auf Beobachtung einer größeren Anzahl Patienten mit adenoiden Vegetationen beruht und dureh systematisches Ausfragen und genaue Untersuchung jedes einzelnen Patienten zustande gebracht worden ist. Einem solehen Ausfragen und einer genauen Untersuchung wird seit einigen Jahren jeder Patient in der Klinik unterworfen, und ftir jeden einzelnen Patienten wird ein Fragebogen mit folgendem Inhalt ausgefullt:

Ist die Nasenrespiration gehindert gewesen? Zeigte sich: Schnarchen? Geschlossenes Näseln? Neigung zu Sehnupfen? Nasenbluten? Herabsetzung des Geruchsinnes? Pavor nocturnus? Ohrenfluf? Ohrenschmerzen? Ohrensausen? Schwerhörigkeit? Neigung zu Angina? Neigung zu Katarrhen der unteren Luftwege? Kopfsehmerzen? Anämie? Enuresis? Aprosexie? Mangelhafte geistige Entwicklung?

Von März 1899 bis Jannar 1902 wurden in der Klinik für Ohren-, Nasen- und Halskrankheiten 500 an adenoiden Vegetationen leidende Patienten behandelt, d. h. 19,2 Proz. unter sämtlichen Patienten der Klinik. Unter diesen 500 Patienten waren 246 männlichen, 254 weiblichen Geschlechts; es zeigte 
Die durch adenoide Vegetationen hervorgerufenen Symptome usw. 187

sich mithin kein bemerkenswerter Untersehied des Auftretens bei den beiden Geschlechtern. Das Durchschnittsalter war für die beiden Geschlechter 9,2 Jahre, für die männlichen Patienten 8,6 , für die weiblichen 9,7 . Im ubrigen verteilten sich die 500 Patienten in folgender Weise auf die versehiedenen Altersstufen.

\begin{tabular}{c|c|c|c} 
Alter & $\begin{array}{c}\text { Männl. } \\
\text { Gesohlecht }\end{array}$ & $\begin{array}{c}\text { Weibl. } \\
\text { Geschlecht }\end{array}$ & Im ganzen \\
\hline Unter 5 Jahren & 36 & 35 & 71 \\
$6=$ & 28 & 16 & 44 \\
$7=$ & 30 & 30 & 60 \\
$8=$ & 31 & 32 & 63 \\
$9=$ & 39 & 28 & 67 \\
$10-14=$ & 72 & 84 & 156 \\
$15-19=$ & 9 & 22 & 31 \\
20 Jahren a. daruber & 1 & 7 & 8 \\
\hline Samtl. Altersstufen & 246 & 254 & 500
\end{tabular}

Aus den Zahlen geht zur Genüge hervor, daß die adenoiden Vegetationen eine "Kinderkrankheit" im eigentlichen Sinne dieses Wortes ist; nach dem vollendeten 15. Lebensjahre treten sie immer seltener auf, und nach dem vollendeten 20. Lebensjahre trifft man sie nur sehr selten an. Unter unsern Patienten war die Altersstufe von 9 Jahren am stärksten vertreten, aber auch die Stufen von 7 und 8 Jahren waren stark repräsentiert, so daß diese 3 jährige Periode mit 38 Proz. unter sämtlichen Patienten am höchsten zu stehen kommt. Was die adenoiden Vegetationen bei erwachsenen Individuen betrifft, so ist die Krankheit unzweifelhaft früher, bevor die Adenotomie eingeführt war, viel häufiger aufgetreten. Den Beleg hierfur findet man in Wilh. Meyers bekannter Abhandlung, im Archiv für Obrenheilkunde Bd. VII und VIII veröffentlicht. M. bearbeitete damals ein Material, welches 175 Patienten mit Vegetationen umfaßte; unter denselben fanden sich nicht weniger als 38, d. h. 21,1 Proz. im Alter von mehr als 20 Jahren. Das vorliegende Material aus unserer Klinik hat für dieses Alter nur 1,6 Proz. aufzuweisen. Die Ehre für dieses günstige Resultat müssen wir W. Meyer zuschreiben; denn die Ursache kann nur in dem. Umstande liegen, daß die Patienten jetzt im frühen Lebensalter zur Behandlung kommen, weil heutzutage die Aufmerksamkeit in so hohem Grade auf das Vorhandensein von adenoiden Vegetationen bei Kindern gerichtet ist.

Die Symptome, Folgekrankheiten und Komplikationen, welche die adenoiden Vegetationen begleiten, sind sehr zahlreich und 
verschiedener Art. Bevor wir auf eine näbere Beschreibung derselben eingehen, wird es deshalb aus praktischen Rücksichten von Vorteil sein, eine schematisehe Ubersicht über die Reihenfolge zu geben, in welcher sie besprochen werden sollen.

I. Symptome, welche infolge von Verschluss im Nasenrachenraume entstehen: Nasenstenose, Anosmie und Rhinolalia clausa.

II. Katarrhalische Affektionen der schleimhäute der Luftwege: Rhinitis acuta catarhalis, Rbinitis chronica hypertroplica, Rhinitis chronica atrophica, Rhinopharyngitisacutacatarrhalis, Rhinopharyngitis chronica catarralis, Pharyngitis acuta et Tonsillitis acuta (Angina), Pharyngitis chronica catarhalis, Tonsillitis chronica, Laryngitis und Bronchitis.

III. Ohrenleiden: Ocelusio tubarum, Otitis media catarralis acuta, Otitis media catarralis chronica, Otitis mediasuppurativa acuta, Otitis media suppurativa chronica.

IV. Ferner liegende Symptome: Epistaxis, Enuresis, Kopfschmerzen, Anämie, Aprosexie, Stottern und Stammeln, mangelhafte geistige Entwicklung.

\section{Symptome, die infolge von Verschluss im Nasenrachenraume entstehen.}

Nasalstenose. Dieselbe wurde bei 424 , d. h. 83,8 Proz., nuter den Patienten konstatiert; sie stellt sich dadureh als das konstanteste unter allen Symptomen heraus. Alle Abstufungen der Nasenstenose sind hier mit gezählt worden, und es wurde nicht darauf Rücksicht genommen, ob das Symptom einen sta. bilen oder einen mehr vortibergehenden Charakter anfwies.

Es liegt die Annahme nicht fern, daß die Nasalstenose ausschließlich darauf berthe, daß die Choanae durch adenoide Massen zugestopft werden. So einfach läBt sich das Symptom doch wohl kaum erklären; denn die Vegetationen müßten in diesem Falle kolossale Dimensionen haben und weit größer sein, als sie in Wirklicheit sind. In vielen Fällen gestalten sich die Verhältnisse zwar so, daß der Luftstrom durch die Nase aus seiner normalen, aufsteigenden Richtung hinausgedrängt wird, wenn die Vegetationen auch nur den oberen Teil der Choanae bedecken; der Luftstrom nimmt alsdann seinen Weg durch den Meatus inferior, welcher, wenn Vegetationen vor- 
banden sind, immer der Verlegung durch Schleimhautschwellung: und durch Sekretmassen ausgesetzt ist; demzufolge entsteht leicht eine bedeutende Nasenstenose, auch dann, wenn die Vegetationen nicht besonders grob sind. Die entscheidende Rolle bei der Entstehung und Entwicklung der Stenose spielen aber selbstverständlich die Größe, der Sitz und die Gestalt der Vegetationen, sowie anch - und dies ist vielleicht das Wesentlichste - die gröBere oder geringere Geräumigkeit des Nasenrachenraumes. Daß die Geränmigkeit des Nasenrachenraumes eine große Rolle spielt, geht daraus hervor, dah wir bei 6 unter unsern Patienten grobe, in einem Falle sogar sehr große Vegetationen fanden, ohne daß dieselben Nasalstenose hervorgerufen hatten.

Die direkte Folge der Nasenstenose ist die $\mathrm{Mundrespi-}$ ration, welche bekanntlich in jeder Beziehung dem normalen Respirationsmodus nachsteht. Besteht die Nasenstenose durch längere Zeit hindurch, und besonders wenn dieselbe im frthen Kindesalter entstanden ist, so entwickelt sich der bekannte adenoide Habitus; wie häufig derselbe auftritt, läßt sich anf statistischem Wege nicht wohl nachweisen, weil man ihn in allen Nuancen von der leisen Andeutung bis zum stark ausgesprochenen Bilde antrifft, so dab die Entscheidung in hohem Grade auf einem Gutachten zu beruhen kommt.

Während des Schlafes werden noch leichter als im wachen Zustande dem freien Luftstrom durch die Nase Hinderungen in den Weg gestellt; denn die liegende Stellung begünstigt die Entstehung von venöser Stase in der Nase und im Nasenrachenraum, und außerdem werden während des Schlafes die freiwilligen Bestrebungen des Patienten, das Sekret aus den erwähnten Kavitäten zu entfernen, eingestellt. Im allgemeinen macht sich beim Anfang des Schlafes ein unbewußtes Streben geltend, die Respiration in normaler Weise vorgehen zu lassen, und dies wird besonders dann der Fall sein, wenn die Nasenstenose während des Tages einen mehr labilen Charakter zeigt. Der schnell sich einstellende Luftmangel zwingt indes das Kind, durch den Mund zu atmen; auf diese Weise können Nasen-und Mundrespiration durch einen längeren Zeitraum abwechseln. Sehließlich fallt dann das Kind in einen ruhigen Schlaf, schläft aber mit offenem Munde, während ein lautes Schnarch en ertönt, welches durch die schlürfenden Bewegungen des Gaumensegels und vielleicht auch durch das Zurüeksinken der Zunge in die Mundhöhle entsteht. Bei nicht weniger als 387 (d. b. 77,4 Proz.) 
unter unsern Patienten war der Schlaf von Schnarehen begleitet.

In einigen Fällen zeigten siob, besonders am Anfang des Schlafes, starke Unruhe und häufiges Aufwachen; oft hat das Kind laut gesehrien, und nicht selten hat es böse Träume gehabt. Indes war nur in bezug auf 100 Patienten die Aufmerksamkeit auf das Vorhandensein eines solchen Pavor noct urnus gerichtet gewesen; derselbe war bei 17 , d. h. 17 Proz., beobachtet worden. Auf die Umgebungen des Kindes kann dieser Zustand sehr beängstigend einwirken, und in einigen Fällen hatte eben dieses Symptom Veranlassung gegeben, daß das Kind unter ärtliche Behandlung kam.

Anosmie. Teilweiser oder vollständiger Verlust des Gernchsinnes ist kein seltener Begleiter der adenoiden Vegetationen; es ist aber nicht ganz leicht, dieses Verhältnis zu untersuchen, weil die Mehrzahl der Patienten Kinder sind, die, wenn es eine Untersuchung des Geruchsinnes gilt, nur sehr unbestimmte Aufklärungen geben können. Auch dann, wenn man verschiedene Riechstoffe in Anwendung bringt, kommt man der Sache nicht näher; die Antworten sind zu unbestimmt, so daß man ihnen keinen Wert beilegen darf. Es ergibt sich denn auch, daf in den meisten Journalen die Fragen, welche dem herabgesetzten Geruchsinn galten, nicht beantwortet werden in bezug auf die kleinen Kinder; erst in bezug anf Kinder im Alter von 10 Jahren findet man Antwort auf diesbezügliche Fragen. Es ist mithin die Annahme wohl erlaubt, daß die Patienten, welche das 10. Lebensjahr zurüekgelegt haben, genügende Aufklärung baben geben können, und deshalb wird unsere Untersuchung nur solche berücksichtigen.

193 unter unsern Patienten waren mehr als 10 Jahre alt, und bei 52, d. b. 26,9 Proz., unter denselben war der Geruchsinn geschwächt oder aufgehoben; ferner konstatierten wir, daß in den Fällen, wo diesbezügliche Aufklärungen vorlagen, der Geruchsinn im Laufe eines kurzen Zeitraumes bedeutend verbessert wurde, nachdem die Adenotomie ausgeführt worden war.

Es ist nun die Frage, wie diese Hyposmie oder Anosmie aufzufassen ist; ist sie essentiell oder respiratorisch? Wilh. M eyer berührt die Frage nur ganz leicht und darf die Anosmie nicht direkt den adenoiden Vegetationen zur Last legen, weil er in den betreffenden Fällen immer einen bedeutenden Katarrh der Nasenschleimhaut vorfand. Die meisten Verfasser betrachten 
Die durch adenoide Vegetationen hervorgerufenen Symptome usw. 191

indes das Vorbandensein eines solehen Katarrhs mehr als Nebensache und geben der Nasenstenose die Schuld. Die in der Klinik gemachten Erfahrungen bestätigen die letztere Annahme. Unter den oben genannten 52 Patienten fanden sich nämlich 22 , bei denen durchans keine Neigung zu Rhinitis nachgewiesen wurde, und deren Nasenschleimhaut bei der Untersuchung naturlicb erschien, wäbrend andererseits sämtliche Patienten mit Ausnahme von zweien eine ausgesprochene Nasenstenose aufwiesen. Außerdem spricht noch die schnell auf die Adenotomie sich einstellende Verbesserung des Geruchvermögens gegen die Annahme, daß die Anosmie auf einem Leiden des Riechepithels berube.

Rhinolalia clausa. Geschlossenes Näseln wurde bei 47 Patienten $(80,3$ Proz.) nachgewiesen; dasselbe tritt mithin als ein so bäufiges Symptom auf, daß das Vorbandensein desselben bei Kindern immer die Aufmerksamkeit anf vorhandene adenoide Vegetationen hinlenken muß. Während die Nasenstenose fast immer sehr schnell nach der Ausfahrung der Adenotomie verschwand, geht aus unsern Journalen hervor, daß dies nieht mit der Rhinolalie der Fall war. Sowohl der "tote" Klang, als auch die mangelhafte Aussprache der Nasenlaute können häufig lange bestehen, verlieren sich aber allmählich nach Ablauf einiger Monate. Wahrseheinlich gewöhnt sich das Kind erst nach und nach an die neuen Verhältnisse und lernt, sein Gaumensegel richtig zu gebrauchen. Es ist desbalb von Wichtigkeit, daß man die Umgebungen der operierten Kinder daranf aufmerksam macht, daß die Kinder nicht immer sogleich, sondern bäufig nur durch besondere Anleitung den Gebrauch der Nasenrespiration erlernen können.

In einem einzelnen Falle trat nach der Operation eine Rhinolalia aperta auf, welche bekanntlich viel mißkleidender ist als das geschlossene Näseln und deshalb bei den Verwandten der Kinder Unzufriedenheit hervorruft. Gliteklicherweise verschwand dieselbe ziemlich schnell, und man darf wohl annehmen, daß dies in den meisten Fällen geschehen wird, weil die Erscheinnng auf einer vorubergehenden, mangelhaften Kontraktion der Pharynxmuskulatur oder einer ebenfalls vortibergehenden Parese des Velum palatinum beruht. Es ist jedoch wohl möglieh, daß das Näseln in gewissen Fällen fortwährend nicht versehwinden will, nämlich wenn sich hinter den adenoiden Vegetationen eine Rhinolalia aperta versteckt hielt und nicht fruher an den Tag 
treten konnte, weil der Luftstrom durch die Nase gehindert war.

\section{Katarrhalische Affektionen der Scbleimhänte der Luftwege.}

Diese Katarrhe bilden ein sehr wichtiges Glied in der Reihe von Symptomen, welche durch die adenoiden Vegetationen hervorgerufen werden; die Disposition fur akute und chronisehe Katarrhe der Schleimhäute der Luftwege ist so hervortretend, daß sie mit wenigen Ausnahmen in der einen oder der anderen Form bei sämtlichen Patienten nachgewiesen werden konnte. Die Ursachen der Katarrhe liegen in der herabgesetzten Widerstandsfähigkeit der Schleimhäute gegen schädliche Einwirkungen; die Schleimhäute der Nase und des Nasenrachenraumes leiden unter der teilweisen oder vollständigen Stockung des Luftstromes durch die Nase, die der tieferliegenden Luftwege unter der Mundrespiration. Von allem andern abgesehen, stellen sieh endlich die Vegetationen mit ihrer schwachen epithelialen Decke als ein für die Infektion stets offenes Eingangstor dar.

Rhinitis acuta catarhalis. Fur 299 (59 Proz.) unter unsern Patienten lagen Aufklärungen dartiber vor, daß sie häufig an "Schnupfen" gelitten hatten. Wahrscheinlich ist doch dieser Prozentsatz zu klein; denn die leichteren Formen der Rhinitis verlaufen nicht selten, ohne von dem Patienten und seiner Umgebung bemerkt zu werden, wie dies bei der objektiven Untersuchung sich mehrmals heransstellte.

Bei einem Drittel unter den Patienten handelte es sieh um eine akute Verschlimmerung einer schon vorhandenen ehronischen Rhinitis.

In 25 Fällen haben wir in der Klinik selbst eine akute Rhinitis beobachtet, und bei der Untersuchung stellte sich heraus, daß gleichzeitig ein Katarrh der angrenzenden Sohleimhäute, besonders der des Mittelohres, auftrat. Chronische Veränderungen der Nasenschleimhaut fanden sich bei 87 Patienten (17,4 Proz.).

Rhinitis chronica hypertrophica wurde bei $54 \mathrm{~Pa}-$ tienten (10,8 Proz.) beobachtet; in vielen Fällen war dieselbe unzweifelhaft dadurch hervorgerufen geworden, daßdie Schleimhant zwischen den schnell aufeinander folgenden akuten Angriffen der Katarrhe keine Ruhe finden konnte. Aber auch der anhaltende Kongestionszustand, in welchem die Nasensehleimhaut sich beim Vorhandensein von adenoiden Vegetationen häufig befindet, spielt hierbei unzweifelhaft eine Rolle. Eine mehr begrenzte und 
Die durch adenoide Vegetationen hervorgerufenen Symptome usw. 199

bedentende Schwellung der Schleimhaut am hintern Ende des Concha inferior fanden wir bei 9 Patienten; in diesen Fällen ist vielleicht die venöse Stase mit im Spiele, aber die Hypertrophie war bei 5 unter den Patienten so konstanter Natur, dab sie nach der Adenotomie mittelst der kalten Sehlinge entfernt werden mußte.

Rhinitis chronica atrophica, die sich vorzugsweise dadurch erkennbar machte, daß die räumlichen Verhältnisse der Nasenkavitäten vergrößert waren, weil die Concha inferior an Umfang verkleinert war, fanden wir bei 33 Patienten (6,6 Proz.). Die Sehleimhant war in sämtlichen Fällen blaß, kollabiert, mit einen sparsamen Sekret bedeckt, welches hin und wieder Neigung zu Borkenbildung zeigte. Nur bei einem Patienten, einem Mädchen von 13 Jahren, ließ sich gleichzeitig ein etwas fötider Geruch spüren.

Rhinopharyngitis acuta catarrhalis ist mit der Pharyngitis acuta so nahe verwandt, dab sie mit dieser zusammen ihre Besprechung finden wird.

Rhinopharyngitis chronica catarhalis. Der Rhinopharynx ist im eigentlichen Sinne die Brutstätte der Katarrhe. Durch das Vorhandensein der adenoiden Vegetationen wird die Schleimhaut hier häufig in einem anhaltenden Kongestionszustand gehalten, die adenoiden Vegetationen begünstigen durch ihre tiefen Spalten und Nischen die Anhäufung von Detritus und Mikroorganismen, und außerdem zeigt das follikulare Gewebe immer entschiedene Neigung zu Entzindung. Beim chronischen Katarrh wird gewöhnlieh eine bedeutende Sekretmenge ansgeschieden, welche über die Pars oralis pharyngis hinabgeleitet; unsere Journale geben keinen genügenden Aufschlub daruber, wie häufig dieses Symptom der Rhinopharyngitis aufgetreten ist.

Pharyngitis acuta et Tonsillitis aeuta (Angina). Es liegt in der Natur der Sache, daß diese Leiden nur ansnahmsweise in der Klinik zur Beobachtung kommen. Die akute Pharyngitis wurde nur $11 \mathrm{mal}$, die akute Tonsillitis $9 \mathrm{mal}$ beobaehtet; in 5 Fällen traten die beiden Leiden gleichzeitig auf. Um feststellen zu können, wie oft diese Katarrhe mit anffallender Häufigkeit bei Patienten mit adenoiden Vegetationen auftreten, richteten wir an alle unsere Patienten oder vielmehr an ihre Umgebungen die Frage, ob sich eine besondere Neigung zu Angina, d. h. akut auftretenden Halsschmerzen in Verbindung mit Fieber und Unwohlsein, bemerkbar gemacht hatte. In dem Sinne, 
wie die Frage sie faßt, umfaßt die Angina mithin sowohl die einfache akute diffuse Pharyngitis und Rhinopharyngitis wie auch die akute Tonsillitis. Es stellte sich heraus, dab die Angina aufgetreten war bei nicht weniger als 159 oder 31,8 Proz. unter den Patienten; wenn aber ca. 1/3 unter den Patienten besondere Neigung zu diesen Katarrhen gezeigt haben, so wird man immer bei Kindern mit häufig recidivierender Angina die Wahrseheinlichkeit von Vorhandensein von adenoiden Vegetationen als Ursache in Betracht ziehen müssen. Zweifellos verlaufen die Prozesse meistens so, daß die akute pharyngeale Tonsillitis den Anfang bildet; nachher werden der Pharynx und die Gaumentonsillen ergriffen. Die beiden ersten Stadien verlaufen häufig unbemerkt, während die dritte Stufe, die palatinale Tonsillitis gewöhnlich diejenige ist, welche zur ärztlichen Behandlung kommt. Interessant ist es zu sehen, daß es Fälle gibt, bei welchen die Angina die alleinige Anzeige davon ist, daß adenoide Vegetationon vorhanden sind. Dies war bei 5 Patienten (1 Proz.) der Fall; dieselben zeigten eine ausgesprochene Neigung zur Angina, während die gewöhnlichen symptome wie Nasenstenose, Naseln u. s. w. feblten. Nachdem die Adenotomie ansgeführt worden war, stellte sich die Angina bei diesen 5 Patienten entweder gar nicht mehr ein oder trat nur äußerst selten bei ihnen auf. Dasselbe Verhältnis, nämlich daß die Neigung zu Angina nach unternommener Adenotomie aufhört, hatten wir bei den ubrigen Patienten mehrmals zu beobachten Gelegenheit.

Pharyngitis chronicacatarhalis. Dieselbe begleitet in der Regel die chronische Rhinopharyngitis in Gestalt einer diffusen Schwellung der Schleimhänte; auf dieses Verhältnis haben jedoch unsere Journale keine weitere Rücksicht genommen. In höherem Grade wurde die Aufmerksamkeit auf den Zustand der Schleimhaut gerichtet, welchen man als Pharyngitis granulosa bezeichnet; dieselbe wurde bei 163 (32,6 Proz.) unter unsern Patienten nachgewiesen. Dieser Prozentsatz gibt indes keineswegs an, wie häufig in Wirklichkeit die Pharyngitis granulosa bei unsern Patienten aufgetreten ist; denn für die Mehrzahl unter den übrigen 337 Patienten liegen keine Aufklärungen über den Zustand der Pharynxsehleimhaut vor. Was die Bezeichnung Pharyngitis betrifft, so ist dieselbe kaum ganz zutreffend; denn die Granulationen zeigen unzweifelhaft nur an, daß das recht sparsame und zerstreute lymphoide Gewebe der 
Pharynxsehleimhaut in die allgemeine Hyperplasie des lymphoiden Gewebes dieser Regionen mit einbezogen worden ist, und die Entwieklung einer solehen Pharyngitis granulosa wird mithin vermutlich auf eben denselben Ursaehen beruhen, die eine Hyperplasie der Pharynxtonsille selbst hervorrufen.

Tonsillitis ehronica. Hyperplasie der Tonsillen wurde bei 108 Patienten (21,6 Proz.) konstatiert. In 51 Fällen tritt diese Hyperplasie mit recidivierender Angina verbunden auf, und es zeigt sich, daf die Tonsillen besonders bei diesen $\mathrm{Pa}$ tienten eine ansehnliehe Größe erreicht hatten; denn bei 22 unter diesen 51 Patienten wurden die Tonsillen entfernt, während dies bei den übrigen 57 Patienten, die keine Neigung zu Angina zeigten, nur 14 mal der Fall war.

Laryngitis. Leider erhalten unsere Journale nur sparsame Aufklärungen über Larynxaffektionen; nur wenn solche sehr hervortretend erschienen, haben sie Erwähnung gefunden. Das vorliegende Material ist mithin nicht hinreichend, wenn es gilt zu untersuchen, wie häufig der Larynx beim Vorhandensein von adenoiden Vegetationen erkrankt und in welcher Weise dies alsdann geschieht.

Besonderes Interesse beansprucht die mit der Larynxstenose verbundene akute Laryngitis und der Laryngospasmus, weil wir bei Kindern dieselben recht häufig mit den adenoiden Vegetationen in Kausalverbindung bringen können. In dieser Beziehung gibt die folgende Krankengesehiehte reoht guten Aufschluss:

Oscar A, 3 Jahre alt. Im ersten Lebensjahre Rachitis. Schon als das Kind einen Monat alt war, traten bei ihm laryngostenotische Anfalle auf, welche im Laufe des letztverflossenen Halbjahres mit größerer Intensität und immer häufiger sich einstellten, so daß sie jetzt sowohl bei Tag als aucb bei Nacht mehrmals anftreten. Gewöhnlich werden diese Anfälle von einem so bedeutenden Stridor begleitet, daß derselbe weithin vernehmber ist, und die Eltern dadurch nachts geweckt werden; gleichzeitig stellt sich starke Cyanose des Angesichts ein. Während des Schlafes schnarcht das Kind fortwährend, und es erwacht fast jede Stunde, indem entweder ein laryngostenotischer Anfall sich einstellt, oder das Kind schreit laut, als sei es durch irgend etwas erschreckt worden. Fast immer findet sich Nasenstenose. Die Sprache ist nãselnd, fast unverständlich. Das Gehör bedeutend herabgesetzt. Enuresis diurna et nocturna mehrmals täglich.

Bei der objektiven Untersuchung werden Zeichen einer uberstandenen Rachitis konstatiert. Das Kind ist schlecht ernäbrt. Es will bei der Untersuchung nicht sprechen. Cavam nasi mit einem mukopurulenten Sekret gefüllt, welches weitere Untersuchung dieser Kavität unmöglich macht; Ohren natülich. Die Tonsillen sind etwas hypertophisch, und im Rhinopharynx fühlen sich große, feste adenoide Vegetationen. Die letzteren entfernt man durch Adenotomie.

Schon in der folgenden Nacht war er Schlaf ungestört; es stellte sich kein Anfall von Larynxstenose ein, und auch später ist kein solcher aufge- 
treten. Enuresis diurna trat nach der Adenotomie nicht wieder auf, die Enuresis nocturna zeigte sich ein paarmal im Ablauf der ersten beiden Wochen nach der Operation, spăter aber nicht wieder. Das Gehör ist verbessert worden, die Sprache ist verständlich. Der Ernăbrungszustand ist verbessert worden. (Observationszeit $1 / 2 \mathrm{Jahr}$ ).

Die Ursachen der Larynxstenose können zweifellos verschiedener Art sein; am bäufigsten bildet aber ohne Zweifel eine akute Sehwellung der Larynxschleimhaut die Ursache; indes ist es auch wohl möglich, daß in einigen Fällen ein auf nervöser Grundlage beruhender, auf dem Wege des Reflexes ausgelöster Krampf die Ursache hergibt, und die angeführte Krankengeschichte gibt vielleicht hierauf ein Beispiel. Die leichteren Stufen der Larynxstenose entstehen vermutlich einfach dadureh, daß hinabfließendes Sekret in den Larynx hineingleitet.

Bronchitis. Ein Mitleiden der tieferliegenden Luftwege wird selbstverständlich leicht eintreten, wenn die Neigung zu Katarrhen so grob ist, wie es hier der Fall ist. Man findet deshalb auch bei diesen Patienten nicht selten sowohl akute wie auch chronische, mit Husten verbundene Formen von Bronohitis. Unser Material gibt aber hieruber nur unvollkommene Aufklärung. Hin und wieder stellt sich ein starker, anhaltender, trockener oder von geringer Schleimabsonderung begleiteter Husten ein, ohne daß eine Laryngitis oder Bronchitis sich nachweisen lassen. Dieser Husten, Halshusten genannt, der sich gewöhnlich abends, gleich nachdem der Patient sich zu Bett gelegt hat, einstellt, kann mitunter wie mit einem Schlage beseitigt werden, wenn die Vegetationen entfernt werden; man ist deshalb wohl anzunehmen berechtigt, daß dieser Husten entweder auf reflektorischem Wege durch die adenoiden Vegetationen, oder auch durch das Hinabgleiten des Schleimes aus dem Nasenrachenraume hervorgerufen wird. Der folgende Fall beleuchtet dieses Verhältnis recht deutlich:

Anna M., 10 Jahre alt, wurde am 30. Januar 1900 in der Klinik vorgestellt. Fast den ganzen Winter hindurch hustete das Kind jede Nacht, sobald es eingeschlafen war, und der Husten dauerte hänfig die ganze Nacht hindurch. Der Husten ist trocken, aber bin und wieder kommt doch etwas Schleim an den Tag. Das Kind leidet fast ununterbrochen an Nasenstenose, hin und wieder an Ohrenschmerzen, mitunter an Nasenbluten. Alle möglichen Mittel gegen den Husten wurden vergeblich versucht. Die im Nasenracbenraume sich findenden weichen, mittelgroßen, kammförmigen Vegetationen entfernt man durch Adenotomie.

In der folgenden Nacht schlief das Kind ruhig. Es atmet durch die Nase und hustet nicht; der Husten stellt sich im Ablaufe der ersten 4 Wocben nur hin und wieder ein, bört aber darnach ganz auf. (Observationszeit $21 / 2$ Jahre.) 
Die durch adenoide Vegetationen hervorgerufenen Symptome usw. 197

\section{Ohrenleiden.}

Bei 322 Patienten (66,4 Proz.) hatten sich angeblich Symptome von seiten des Ohres gezeigt. Die einzelnen Symptome verteilen sich in folgender Weise: Sehwerhörigkeit zeigte sich in 226 Fällen (57,2 Proz.), Ohrenschmerzen in 170 (34 Proz.), Ohrensausen in 131 (26,2 Proz.) und Ohrenfluß in 102 (10,4 Proz.) Alle Symptome miteinander traten bei 35 Patienten ( 7 Proz.) auf, während 83 (16,6 Proz.) nur an Sehwerhörigkeit, 15 (3 Proz.) nur an Ohrenschmerzen, 10 (2 Proz.) nur an Ohrensausen und 6 (1,2 Proz.) nur an Obrenflub gelitten hatten. Ganz zuverlässig sind diese anamnestisehen Mitteilungen wohl nicht. Die gefundenen Prozentsätze sind doch wahrscheinlich eher zu niedrig als zu hoch; denn leichtere Stufen z. B. von Schwerhörigkeit werden häufig weder von dem Patienten noch seiner Umgebung bemerkt; die Tatsache aber, dab nicht weniger als zwei Drittel unter den Patienten angeblich Symptome von seiten des Ohres zeigten oder gezeigt hatten, genügt, um uns zu tberzeugen, daß zwischen diesen Affektionen und den adenoiden Vegetationen ein intimes Verhältnis besteht. Sobald deshalb ein Kind wegen Ohrenleidens zur ärztlichen Behandlung kommt, mub der Arzt immer seine Aufmerksamkeit auf eine eventuelle Hyperplasie der Pharynxtonsille richten.

In Bezug auf die verschiedenen Formen der Obrenleiden ergab die objektive Untersuchung folgendes Resultat:

Occlusio tubarum. Tubaverschluß wurde in 91 Fällen (18,2 Proz.) nachgewiesen; es wurde bei diesen Patienten deutliche Retraktion der Trommelfelle beobachtet, ohne daß gleichzeitig katarrhalische Veränderungen vorhanden waren.

Otitis media catarhalis acuta. Dieses Ohrenleiden wurde 16 mal (3,2 Proz.) gefunden, bei 10 Patienten war dasselbe einseitig, bei 6 doppelseitig; in 12 Fällen handelte es sich um eine Exacerbation eines vorhandenen chronischen Prozesses. An und für sich haben die gefundenen Prozentsätze keine große Bedeutung, weil es in der Regel ganz zufällig ist, wenn die Patienten gerade an einem Zeitpunkte zur Behandlung kommen, wo nachweisbare katarrhalische Veränderungen des Mittelohrs vorhanden sind. Wollen wir die Häufigkeit, mit welcher unter diesen Verhältnissen akute Katarrhe des Mittelohrs auftreten, richtig schätzen lernen, so müssen wir die früher erwähnten, von den Patienten angegebenen ansehnlichen Prozentsätze für Ohrensehmerzen und Ohrensausen in Betracht ziehen; denn diese 
beiden Symptome der katarrhalisehen Veränderungen des Mittelohres sind gerade diejenigen, die von dem Patienten selbst und seiner Umgebung bemerkt werden, während die auch begleitende Schwerhörigkeit häufig sich der Beobachtung entzieht.

Otitis media catarhalis chronica wurde bei $56 \mathrm{~Pa}$ tienten beobachtet $(18,2$ Proz.); in 46 Fällen waren beide Ohren, in 10 Fällen nur das eine Ohr affiziert; unter den letztgenannten 10 Patienten fand sich in 6 Fällen am anderen Ohre eine chronische suppurative Mittelohrentzündung. Die Trommelfelle stellten sich gewöhnlich verdickt, meistens glanzlos und weiß oder grau gefärbt dar; ein paarmal wurden Kalkablagerungen beobachtet, verbältnismäßig selten, nämlich nur bei 9 Patienten, waren die Trommelfelle deutlich atrophisch.

0 titis media suppurativa a cuta wurde bei 7 Patienten (1,4 Proz.) konstatiert; bei 3 war das Leiden rechtsseitig, bei 2 linksseitig und bei 2 beiderseitig. Paracentese des Trommelfells wurde 2 mal unternommen; in den übrigen Fällen hatte sich das Sekret spontan Ablauf geschaffen.

Otitis media suppurativa chronica. Bei 32 Patienten (6,4 Proz.) fanden sich Eiterungen des Mittelohres, welehe schon durch einen längeren Zeitraum bestanden hatten; bei 9 war das Leiden rechtsseitig, bei 11 linksseitig, bei 12 beiderseitig. In fast sämtlichen Fällen war das Sekret stark tubelriechend.

Auber diesen 39 Patienten, bei welchen in der Klinik selbst Eiterungen des Mittelohrs beobachtet wurden, fanden sich noch 23, bei welchen deutliche Zeichen einer überstandenen Mittelohreiterung vorhanden waren. Der objektive Befund war in 16 Fällen größere oder geringere Defekte des Trommelfells, in 7 Fällen Cicatricen des Trommelfells. Laut der anamnestischen Aufklärungen hatten indes 102 Patienten an Obrenfluß gelitten, und es bleiben mithin 40 Patienten íbrig, bei denen die Spuren des überstandenen Prozesses vernichtet waren.

Wie aus dem obigen hervorgeht, erstrecken sich die Ohrenleiden, welche die adenoiden Vegetationen begleiten, über das ganze Gebiet ron dem leiehtesten Tubenkatarrh bis zu der schwersten Eiterung des Mittelohrs, und wir sind mithin nicht imstande, aus der Art des Ohrenleidens einen SchluB auf die Kansalverbindung zu machen. Auf zweifache Weise wird auf das $0 \mathrm{br}$ eingewirkt, teils durch Druck an das Ostium pharyngeale tubae, teils durch Fortpflanzung eines Katarrhs des Nasenrachenraumes. In Bezug auf das erstgenannte Ver- 
Die durch adenoide Vegetationen hervorgerufenen Symptome usw. 199

hältnis wird die Größe der Vegetationen von Wichtigkeit sein, aber im übrigen finden wir, daß auch kleine Vegetationen sogar ein schweres Ohrenleiden hervorrufen können, indem die Lokalisation des adenoiden Gewebes, die Rhinopharyngitis und die eventuell vorhandene Disposition hierbei unzweifelhaft bervortretenden Einfluß ausïben. Man kann deshalb Falle antreffen, bei denen die Hyperplasie des adenoiden Gewebes so gering ist und die räumlichen Verhältnisse des Nasenrachenraumes so günstig, daß die allgemeinen adenoiden Symptome: Nasenstenose, Näseln u.s.w. fehlen, wo aber trotzdem ein vorhandenes Ohrenleiden durch Vegetationen hervorgerufen worden ist, deren Gegenwart man erst bei der Digitalexploration erkennt. Bei nicht weniger als 16 Patienten (3,2 Proz.) fanden wir ein derartiges Krankheitsbild; ein Ohrenleiden wurde konstatiert, aber keins der gewöhnlichen Symptome der adenoiden Vegetationen war vorhanden. Die Kausalverbindung gibt sich jedoch durch den günstigen Verlauf des Ohrenleidens nach der Adenotomie deutlich zu erkennen.

IV. Fernerliegende Symptome.

Epistaxis. Hänfig auftretendes und mitunter auch starkes Nasenbluten wurde bei 53 Patienten (10,6 Proz.) gefunden. Dieser Prozentsatz ist viel gröber als der, welchen andere Statistiken aufzuweisen haben; denn diese haben gewöhnlich nur 3-4 Proz. zu verzeichnen. Einige Verfasser wollen sogar nicht die Epistaxis mit unter đie Folgen der adenoiden Vegetationen zählen, unzweifelhaft jedoch mit Unrecht. Zwar kann man nicht behaupten, dab jedes "habituelle Nasenbluten," welches bei Individuen mit adenoiden Vegetationen auftritt, mit den Vegetationen in Kausalverbindung steht; in den Fällen aber, wo nach der Beseitigung der Vegetationen das Nasenbluten nach Ablauf einer kurzen Zeit aufhört, darf man wohl mit Sicherheit auf eine Kausalverbindung schließen. Leider hatten wir nicht die Gelegenheit, das Schicksal sämtlicher 53 Patienten nach der Adenotomie zu verfolgen; nur in bezug auf 21 unter ihnen besitzen wir diesbezügliche Aufklärungen. Für dieselben ergaben sieh folgende Resultate: Bei 15 hörte das Nasenbluten vollständig auf, bei 3 stellte es sich weit seltener ein, und nur bei 3 dauerte es unverändert fort.

Aus dem unten mitgeteilten Krankenbericht geht die Abhängigkeit des Nasenblutens von den adenoiden Vegetationen mit uberzengender Deutlichkeit hervor: 
Anna A., 13 Jahre alt, litt von ibrer ersten Kindheit an fortwährend an einer mit wechselnder Intensităt auftretenden mangelhaften Luftpassage durch die Nase und an Nasenbluten, welches sich häufig und ohne nachweisbare Veranlassung einstellte und mitunter sehr anhaltend war. Sie beschwert sich ausserdem über Stirndruck. Durch die Untersuchung wurden eine diffuse Hyperplasie der Schleimhäute der beiden Conchae inferiores mit starker Blutüberfüllung sowie auch einige niedrige Kämme von adenoiden Vegetationen im Fornix rhinopharyngis nachgewiesen. Da man der Meinung war, daß das Hauptleiden der Patientin auf der Affektion der Nasenschleimhaut beruhte, so behandelte man die beiden Conchae inferiores mit Acid. trichloracet

Dadurch wurde auf eine kurze Zeit die Nasenrespiration wieder hergestellt, aber das Nasenbluten bestand unverăndert fort. 4 Monate nach der ersten Behandlung berichtet unser Journal über die Patientin wie folgt: Seit einiger Zeit wieder mangelhafte Nasenrespiration; das Nasenbluten tritt häufig und $z$ war mit $Z$ wischenräumen von $2-3$ Wochen auf, ist besonders scblimm während der Nacht und erschöpft die Patientin sehr. Die Nasenschleimhaut erscheint blutüberfüllt, der Zustand des Nasenrachenraumes ist wie bei der vorigen Untersuchung.

Aden ot omie. Es wurden dabei nur kleine Vegetationsmasssen entfernt. Nach der Operation hörte das Nasenbluten wie mit einem Schlage auf, die Luftpassage durch die Nase wurde frei, und der Stirndruck verschwand. Die Heilung hat sich gehalten. Observationszeit 3 Jahre.

In denjenigen Fällen von adenoiden Vegetationen, wo man die habituelle Epistaxis in Verbindung mit chronischer Rhinitis antrifft, wird man natürlich geneigt sein, die Ursache des Nasenblutens in der letzteren Affelktion zu suchen; was den hypertrophisehen Katarrh betrifft, so wird bier die starke Überfullung des eavernösen Gewebes eine Disposition zu Nasenbluten hervorrufen, und bei der atrophischen Form der Rhinitis kann Nasenbluten entstehen, wenn die kleinen Borken sich lösen. Daß dennoch die adenoiden Vegetationen die Hauptrolle spielen können auch in denjenigen Fällen, wo ein Leiden des Cavum nasi vorliegt, das geht aus dem oben beschriebenen Falle deutlich hervor.

Bei 16 Patienten mit häufig auftretendem Nasenbluten ließen sich keine Spuren von pathologischen Veränderungen der Nasenschleimhaut nachweisen, wir sind mithin in diesen Fällen wie in dem oben beschriebenen - darauf angewiesen, die Ursachen des Nasenblutens in Verhältnissen zu suchen, die von der Gegenwart der adenoiden Vegetationen unmittelbar abhängig sind, und hier wird der Verdacht besonders auf eine Venenstase hingelenkt werden müssen. A priori wird das Vorhandensein einer solchen als zweifelhaft erscheinen wegen der vielen Kollateralbahnen, die sich in der Nase finden; aber es ist trotzdem keine Seltenheit, daß schlechte Zirkulationsverhältnisse in der Nasenregion durch adenoide Vegetationen hervorgerufen werden. Einige Patienten beschwerten sich tiber einen unangenehmen 
Die durch adenoide Vegetationen hervorgerufenen Symptome usw. 201

Druck an der Nasenwurzel, und bei der Untersuchung wurde mehrmals eine Schwellung und Rötung der Integumente der Nasenwurzel und an beiden Seiten gefunden, welche sich unter den Margo infraorbitalis hin erstreckte; daß diese Erseheinung durch eine Venenstase hervorgerufen wurde, geht deutlieh daraus hervor, daß sie bei der Beseitigung der Vegetationen alsbald verschwand. Erwähnt sei an dieser Stelle noch der Umstand, daB wir bei einem Patienten mit Epistaxis Venenektasien am Septum fanden; jedoch kann diese Erscheinung sehr wohl auf Ursachen anderer Art beruhen und braucht nicht auf einer durch adenoide Vegetationen hervorgerufenen Stase zu fußen. Es liegt die Annahme nicht fern, dab die Chancen für Zirkulationsstörungen um so größer werden, je größer die Vegetationen sind; daß aber auch kleine Vegetationsmassen in dieser Beziehung Einfluss ausüben können, ist recht erklärlich, weil hier mehrere Verbältnisse in Betracht gezogen werden müssen, nämlich die räumlichen Verhältnisse des Nasenrachenraumes, der Sitz und die Gestalt der Vegetationen und vor allen Dingen die Konsistenz derselben; denn wenn die Vegetationen weich und mit sparsamem Bindegewebe versehen sind, so tritt leicht eine Volumenvergrößerung ein. Die oben mitgeteilte Krankengeschichte liefert uns auch den besten Beweis dafür, daß eine verhältnismäBig kleine Vegetationsmasse. unter gewissen Umständen die unmittelbare Erzeugerin der Epistaxis sein kann; das Nasenbluten trat, wie erwähnt, besonders in der Nacht auf, mithin wenn die Patientin eine liegende Stellung einnahm, und das heibt wieder au einem Zeitpunkt, wo die Pharynxtonsille am stärksten kongestioniert ist und demzufolge ihren größten Umfang hat.

Es ist jedoch die Möglichkeit nicht ganz ausgeschlossen, $\mathrm{daB}$, das Bluten in einigen Fällen gar nicht von der Nasenschleimbaut herstammte, sondern von den Vegetationen selbst. Bekanntlich sind dieselben zu Blutungen sehr geneigt, und hin und wieder kann man, wie schon von W. Meyer bemerkt, bei der Inspektion Blut in der Rachenhöble antreffen (was den Argwohn auf Phthisis erregt); gelegentlich könnte dann wohl auch dies Blut sich einen Ausgang durch die Nase bilden.

Enuresis. Diese Komplikation wird an dieser Stelle nur in aller Kürze Erwähnung finden, weil schon aus unserer Klinik eine Untersuchung vorliegt ${ }^{3}$ ), welche die adenoiden Vegetationen

1) Ugeskrift for Lăger. 1901. Nr. 38 u. 39. 
des Nasenrachenraumes als Ursache der Enuresis betrifft; das Material, welches dieser Untersuchung zu Grunde lag, ist teilweise das nämliche, auf welchem auch die vorliegende Untersuchung beruht. Die Resultate der oben erwähnten Untersuchung waren folgende:

1) Die Enuresis ist eine so häufige Begleiterin der adenoden Vegetation, dab diese Tatsache allein den Schluß berechtigt, daß sie eine durch die Vegetationen hervorgerufene Folgekrankheit sei. Diese Anschaunng wird ferner dureh den Umstand gestiitzt, daß 2) die Enuresis vorzugsweise solche adenoide Vegetationen begleitet, welche mit bedeutender Nasenstenose verbunden sind. Am entscheidendsten spricht jedoch fur die Kausalverbindung die Erscheinung, daß 3) die Enuresis in einer großen Anzahl von Fällen durch die Beseitigung der Vegetationen entweder geheilt oder doch verbessert wird.

Diese Konklusionen wurden auf Grundlage von 716 Fällen von adenoiden Vegetationen aufgestellt; dieselben verteilen sich auf 2 Gruppen, nämlich auf eine aus 400 Patienten aus Professor Myginds Privatklinik und eine aus 316 Patienten der Klinik des Kommunehospitals bestehende Gruppe.

Innerhalb der erstgenannten Gruppe fanden sich $31, \mathrm{~d}$. h. ca. 8 Proz., die zur Zeit der Einleitung der Behandlung die Komplikation der adenoiden Vegetationen mit Enuresis aufwiesen. 26 unter diesen Patienten, die sämtlieh im Alter zwischen 2 und 11 Jahren standen, wurden durch einen längeren Zeitraum hindurch der Beobachtung unterworfen. Es litten 20 unter denselben an Enuresis nocturna allein, 3 ausschließlich an Enuresis diurna, während die tibrigen 3 an beiden Affektionen in Verbindung litten. Die Beseitigung der adenoiden Vegetationen resultierte in Bezug auf diese Gruppe darin, daß 14 (54 Proz.) geheilt wurden, bei 8 (31 Proz.) verbesserte sich das Leiden, bei 4 (15 Proz.) ließ sich keine Veränderung spüren.

Unter der zweiten Gruppe, welche 316 in der Klinik des Kommunehospitals behandelte Patienten umfaßt, wurde die Enuresis bei 75 Individuen nachgewiesen (ca 23 Proz.). Es gelang uns, das fernere Schicksal der 45 Patienten zu verfolgen, unter welchen 40 an Enuresis nocturna allein, 1 an Enuresis diurna und 4 an beiden Affektionen miteinander in Verbindung litten. Die Patienten standen sämtlich im Alter zwischen 3 und 15 Jahren. Das Operationsresultat stellte sich in Bezug auf die Enuresis in folgender Weise heraus: 29 (62 Proz.) wurden von 
Die durch adenoide Vegetationen hervorgerufenen Symptome usw. 203

dem Leiden geheilt, bei 15 (ca. 35 Proz.) besserte sich dasselbe mehr oder weniger, und nur bei einem einzigen Patienten ließ sich gar keine Verbesserung spüren.

Fassen wir sämtliche Patienten unter eine Gruppe zusammen, haben wir es mithin mit 716 Fällen von adenoiden Vegetationen zu tun, unter welchen 106 oder 14,8 Proz. mit Enuresis kompliziert waren. 71 wurden einer fortgesetzten Beobachtung unterworfen, und unter denselben trat in 66 Fallen (93 Proz.) Heilung bezw. Verbesserung des Leidens ein.

Die Erfahrungen, die wir seit dem Erscheinen der obigen Untersuchungsresultate in der Klinik machten, haben in keiner Beziehung diese Konklusionen angefochten. Wie damals, so fanden wir auch jetzt, teils daß die Enuresis häufig mit den adenoiden Vegetationen in Verbindung auftritt, teils daß die Beseitigung der Vegetationen einen günstigen Einfluß auf die Enuresis ausübte.

Als Kuriosum sei an dieser Stelle erwähnt, daß ein 10 jähriges, an Enuresis nocturna leidendes Mädchen in der Klinik vorgestellt wurde, um wo möglich von ihrem Leiden geheilt zu werden. Die Mutter des Kindes hatte nämlich durch Gespräch mit ihren Nachbarsleuten in Erfahrung gebracht, daß mehrere unter den in der Klinik behandelten Kindern von ihrer Enuresis befreit worden waren.

Weder die Examination noch die Inspektion ließen vermuten, daß adenoide Vegetationen vorhanden seien; bei der Digitalexploration wurde aber eine mittelgroße Hyperplasie der Pharyuxtonsille nachgewiesen. Nachdem die Adenotomie ausgefuhrt worden war, hörte die Enuresis mit einem Schlage auf. Natürlich kann in diesem Falle der psychisehe Einfluh auf das günstige Resultat nioht ausgeschlossen werden.

Kopfschmerzen haben bei unsern Patienten sehr hänfig: die adenoiden Vegetationen begleitet; nicht weniger als 221, d. h. 44,2 Proz., hatten daran gelitten. Im allgemeinen waren in diesen Fällen die Vegetationen von mittlerer Größe oder noch größer. Die Schmerzen lokalisierten sich am häufigsten am Nacken oder an der Stirn, nicht selten aber auch ausschließlich an der Glabella; mitunter gaben sie sich als Druck an der Nasenwurzel zu erkennen.

Wahrscheinlich spielt die Anämie, welche bäufig als die Begleiterin der adenoiden Vegetationen auftritt, nur eine untergeordnete Rolle inbezug anf die Entstehung der Kopfschmerzen; 
denn bei 149 (66 Proz.) unter den 221 Patienten fand sich keine Blutarmut. In der Mehrzahl der Fälle müssen mitbin die Ursachen in anderen Verhältnissen gesucht werden, vielleicht in dem mangelhaften AbfluB des Blutes und der Lymphe aus dem Gehirn. Die Venen stehen bekanntlich durch die Venae ethmoidales mit den Venen der Dura mater und der Sinus longitudinalis superior et inferior in Kommunikation, und auch die Verbindung zwischen den Lymphbahnen der Nasensehleimhant einerseits und dem subarachnoidalen Raume andererseits ist eine Tatsache. Katarrhalische Schwellung der Nasenschleimbaut und Venenstase - inbezug auf deren Auftreten man, wie früher erwähnt, einen unmittelbaren Einfiub der Vegetationen nicht ausschließen kann - werden auf die Passage der Lymphe dureh die Nasenschleimhaut und auf den freien Lauf des venösen Blutes durch das kavernöse Gewebe hindernd einwirken.

Ist die Annahme nun richtig, daß die Kopfschmerzen vorzugsweise auf einem erhöhten Druck in den Lymphgefäßen und den Venen beruhen, so werden dieselben selbstverständlich besonders bei solchen Patienten auftreten, deren Nasenstenose bedeutend, und deren Neigung zu Rhinitis hervortretend ist; dies ist auch wirklich der Fall. Bei 112 Patienten konstatierten wir bei der objektiven Untersuchung deutliche Zeichen eines Leidens der Nasenschleimhaut, und unter diesen Patienten gaben 76 (67,9 Proz.) an, daß sie an Kopfschmerzen litten. Unter unsern 500 Patienten fanden wir bei 59,8 Proz. ausgesprochene Neigung zu Rhinitis, aber unter den 221 mit häufig auftretenden Kopfschmerzen hatten 71,7 Proz. Neigung zu dieser Affektion. Vielleicht spielt auch die Nasenstenose eine ätiologische Rolle bei den Patienten, die an Kopfsehmerzen litten, obschon die Nasenstenose nicht viel häufiger $(87,3$ Proz.) unter diesen als unter sämtlichen Patienten nachgewiesen wurde (83,8 Proz).

Anämi e. Hervortretende Blutarmut fanden wir bei $171 \mathrm{~Pa}-$ tienten (34,2 Proz.). Bei 7 Patienten war die Anämie sogar so stark, daß man Bedenken hegte, sie dem mit der Operation verbundenen Blutverluste auszusetzen. Die Adenotomie wurde in diesen Fällen 4-6 Wochen ausgesetzt, und mittlerweile wurde eine Behandlung mit Eisenpräparaten versucht.

Mehrere Umstände tragen dazu bei, daß sich bei der Gegenwart von adenoiden Vegetationen leicht anämische Zustände einstellen. Die Nasenstenose bewirkt, daf die Respiration vorzugsweise durch den Mund gesehieht; dadurch wird der Vorgang 
Die durch adenoide Vegetationen hervorgerufenen Symptome usw. 205

des Atmens oberfläehlich, das Blut wird nur unvollkommen mit Sauerstoff versehen, und die Verbrennungsprodukte werden nur mangelhaft ausgeschieden. Ferner wird durch den unruhigen Schlaf mit den wiederholten kleinen Ersticknngsanfällen das Blut der Überfullung mit Kohlensäure ausgesetzt. Am häufigsten ist das Kind tagsüber müde, mutrrisch und indolent; es hat keine Lust, mit den Kameraden im Freien zu spielen, und besonders hieruber beklagen sich die Eltern. Mitunter ist der Appetit wegen der Trockenheit der Zunge und des Rachens schlecht, und es stellen sich hin und wieder dyspeptisehe Zustände ein, die vielleicht durch die versehlnckten groben Sekretmassen aus dem Nasenrachenraume hervorgerufen werden. Sämtliche oben erwähnten Umstände bewirken, daß die Entwickelung des Kindes unter sehr schlechten Verbältnissen geschieht, weshalb auch Blutarmut und schlechte Ernährungszustände recht häufig als Folgezustände auftreten müssen.

Einige unter unsern Patienten hatten deshalb auch ein kränkliches Aussehen, und bei 5 (1 Proz.) fanden sich hervortretende Zeichen auf Skrophulose. Auffallend viele unter den Patienten hatten nicht die Körperhöhe erreicht, welche Kinder ihres Alters sonst aufweisen. Häufig war der Brustkorb schmal und zugleich flach; letzteres war besonders bei den älteren Patienten hervortretend.

Um eine Beobachtung der Verhältnisse der einzelnen Symptome und des konstitutionellen Zustandes nach der Adenotomie zu ermöglichen, haben wir es unsern Patienten zur Pflicht gemacht, sich ein Paar Monate nach der Operation in der Klinik einzustellen, und die meisten unter ihnen haben dieser Ordre Folge geleistet. Die überwiegende Mehrzahl der Patienten hatten ein weit frischeres und gesunderes Aussehen aufzuweisen, obschon im allgemeinen keine besondere Behandlung angewandt worden war; ganz von selbst haben die Eltern, wenn die Observation sich taber einen längeren Zeitraum erstreckte, mitgeteilt, daß sie ein starkes Wachstum beim Kinde gespürt hatten.

A prosexie. Während der letzten 15 Jahre ist die Aufmerksamkeit auf die Herabsetzung der cerebralen Funktionen als ein Glied in der Symptomatologie der adenoiden Vegetationen gerichtet gewesen. Die Manifestationen der Aprosexie sind folgende: 1. Sohwierigkeit bei der Erwerbung und Assimilation neuer Kenntnisse, besonders wenn dieselben abstrakte Verhältnisse betreffen; 2. Schwierigkeit beim Behalten der erworbenen 
Kenntnisse, mithin Mangel an Gedächtnis und 3. Schwierigkeit bei der Konzentration der Aufmerksamkeit auf einen bestimmten Gegenstand. Die Aprosexie besteht mithin in einer an ein begrenztes Gebiet gebundenen Herabsetzung der Hirntätigkeit und steht in keiner Verbindung mit der Entwicklungsstufe der geistigen Vermögen.

Unseren sämtlichen Journalen zufolge wurde die Aprosexie bei 179 Patienten (35,8 Proz.) nachgewiesen; hieran muissen jedoch einige Bemerkungen angekntipft werden. Will man nämlicb, unter Vermeidung der schlimmsten Fehlschltisse, untersuchen, wie häufig die Aprosexie aufgetreten ist, so wird man nicht sämtliche Patienten mitzählen können; denn bei ganz kleinen Kindern wird man in Wirklichkeit keine solche an besondere Gebiete gebundene Herabsetzung der Hirntätigkeit konstatieren können. Deutlicher wird dagegen die Aprosexie ans Licht treten, wenn das Kind in die Schule eintritt, woselbst es in einen sowohl umfassenden als auch verschiedenartigen Stoff eingefuhrt werden soll, der teilweise rein abstrakter Art ist. Bei der Untersuchung muls man deshalb alle Patienten ausschlieben, die nicht das schulpflichtige Alter von 7 Jahren erreicht hatten, und nur diejenigen mitzählen, welche 8 Jahre und darïber alt waren. Unter unseren Patienten fanden sich 325 solche, und unter denselben hatten 132 (40,1 Proz.) Zeichen von Aprosexie anfzuweisen. Das Geschlecht scheint keinen bemerkenswerten Einfluß in dieser Beziehung auszuüben; jedoch sei es erwähnt, daß unter 10 männlichen Patienten im Alter von mehr als 15 Jahren kein einziger an Aprosexie litt, während unter 29 weiblichen Patienten der selben Altersstufe diese Komplikation bei 8 nachgewiesen wurde.

Die folgende Krankengesehichte zeigt ein typisches Bild auf Aprosexie, dureh adenoide Vegetationen hervorgerufen.

Waldemar 0 ., 9 Jahre alt, leidet seit ein paar Jahren an ziemlich ansgesprochener Nasenstenose und Neigung zin Katarrhen der Nase und der Rachenhöhle. Seine Aussprache ist näselnà, er schläft mit offenem Munde resp. schnarcht nicht wenig. Er beschwert sich über Kopfschmerzen und Müdigkeit, bat auch selten Lust zu spielen. Die Mutter ist der Meinung, $\mathrm{da \beta}$ ihm an Verstand nichts fehle; trotzdem folgt er in der Schule nur schlecht dem Unterricht. Er ist fleißig, bereitet sich lange auf seine Lektionen vor, lernt aber dieselben nur mit Schwierigkeit und hat im allgemeinen die Lektionen vergessen, wenn er examiniert wird. Das Gehörvermögen ist etwas herabgesetzt, bald mehr bald weniger. Häufig tritt Enuresis nocturna auf. Keine nachweisbare Anämie. Bei der Digitalexploration fühlen sich mittelgroße, recht weiche, kammförmige Vegetationen im Rhinopharynx an.

Adenotomie. Etwa 2 Monate spăter erklärt die Mutter, daß die Nasenstenose beseitigt ist; das Gehörvermögen hat sich verbessert, und die Enuresis hat aufgehört. Er ist mehr lebhaft, lernt seine Lektionen viel 
Die durch adenoide Vegetationen hervorgerufenen Symptome usw. 207

leichter als früher, bat in der Schule guten Fortgang gezeigt und ist rom Lehrer zur Überführung in eine böhere Klasse empfohlen worden.

Die Aprosexie tritt bei diesem Patienten sehr deutlich an den Tag, er lernt mit Schwierigkeit und vergißt schnell das Gelernte, und trotz seinem Fleiße kann er den Wettlauf mit den Schulkameraden nicht aufnehmen. Um in Erfahrung zu bringen, welche Unterrichtsgegenstände diesen Schülern der Volkssehule - denn um solche handelt es sich hier - besondere Schwierigkeiten darbieten, haben wir, so oft sich Gelegenheit darbot, diesbezïgliehe Fragen an die Eltern gestellt. Im allgemeinen scheinen die Kinder das Rechnen recht gut aufzufassen, und eben auf diesem Punkt wäre man geneigt, die Schwierigkeiten zu vermuten; dagegen zeigt es sich, dab die Geschichte und die Geographie, mithin diejenigen Disziplinen, welche vorzugsweise an das Gedächnis Ansprtiche stellen, die größten Schwierigkeiten darbieten.

In Bezug auf die Anschauungen tiber die Verhältnisse, welche die Aprosexie bedingen, gehen die Meinungen weit auseinander. Um wo möglich mittels des vorliegenden Materials die Frage etwas zu beleuchten, untersuchen wir an dieser Stelle die Komplikation der Aprosexie mit anderen Folgezuständen welche möglicherweise einen Einfluß ausüben könnten, nämlich die Komplikation mit Nasenstenose, Schwerhörigkeit und Anämie.

Am häufigsten finden wir die Aprosexie von Nasenstenose begleitet, nämlich in 118 unter den erwähnten 132 Fällen $(89,4$ Proz.); etwas seltener tritt die Schwerhörigkeit als Begleiterin der Aprosexie auf, nämlich bei 83 Patienten $(61,4$ Proz.); bei 41 Patienten (31,1 Proz.) war die Aprosexie mit Anämie kompliziert.

Wenn man sich die Aprosexie als eine Folge derjenigen Indolenz und Müdigkeit vorstellt, welche häufig bei blutarmen Kindern zu bemerken ist, so wird dieser Vorstellung dureh die Tatsache widersprochen, daß bei 65,8 Proz. unter den Patienten mit Aprosexie keine Anämie nachgewiesen werden konnte; im ganzen fanden sich verhältnismäBig nicht beträchtlich mehr $\mathrm{Pa}$ tienten mit Anämie unter den an Aprosexie leidenden $132 \mathrm{~Pa}-$ tienten im Alter von mehr als 8 Jahren, als unter unsern sämtlichen 500 Patienten, nämlich 34,2 Proz. gegen 31,1 Proz. Bezüglich der Sehwerhörigkeit ist ein ähnliches der Fall; dieselbe wurde bei 57,2 Proz. unter sämtlichen Patienten, bei 61,4 Proz. unter den an Aprosexie leidenden Patienten gefunden. Wenn man, wie dies von verschiedener Seite gemacht wird, die Schwer- 
hörigkeit als das eigentliehe Kausalmoment bei der Aprosexie aufstellen will, so müssen wir in Bezug auf ein Drittel unter unsern Patienten uns naeh einer andern Erklärung umsehen; denn bei diesen Patienten war das Gehör gut. Ferner ist in Betracht zu ziehen, daß die Schwerhörigkeit in vielen Fällen teils einen sehr labilen Charakter zeigte, teils so geringftugig war, daß ihr Einfluß auf die cerebralen Funktionen des Kindes nur verschwindend klein werden konnte. Wenden wir uns nun an die Nasenstenose, so treten uns hier mehrere Umstände entgegen, welche uns dazu berechtigen, dieselbe an die Spitze zu stellen, wenn es sich darum handelt, die Ursachen der Aprosexie ausfindig zu machen. Fưr die Annahme eines Kausalverhältnisses zwischen diesen beiden Faktoren spricht erstens der Umstand, daß sie so häufig miteinander in Verbindung auftreten, so dab 118 oder 89,4 Proz. unter den 132 Patienten mit Aprosexie an Nasenstenose litten; unter sämtlichen Patienten haben wir indes die Nasenstenose bei 83,3 Proz. gefunden, und da nun die Steigerung des Prozentsatzes in Bezug anf die Patienten mit Aprosexie keine anffallend bedeutende ist, so durfen wir den obigen Zahlen kaum eine besondere Bedentung zuschreiben. Was aber besonders für die Aufstellung der Nasenstenose als Hauptfaktor bei der Entstehung der Aprosexie spricht, ist die oft erstaunliche Schnelligkeit, mit welcher die letztere nach der wohl ansgeführten Adenotomie verschwinden kann, und die mitunter recht plötzlichen Schwankungen in der Intensität der Aprosexie, welche auf die labilen Formen der Nasenstenose zurückgefubrt werden können. Ferner findet sich eine bedeutende Übereinstimmnng zwischen der Aprosexie einerseits und der voribbergehenden geistigen Mudigkeit andererseits, welche Patienten mit akuter Rhinitis ftuhlen können, und auch bei jungeren Individuen mit chronischer hypertrophischer Rhinitis kann man Zustände antreffen, die in vielem an die mit adenoiden Vegetationen in Verbindung auftretende Aprosexie erinnern.

In Bezug auf die Weise, auf welche die Nasenstenose eine Aprosexie hervorruft, sind mehrere Mögliehkeiten vorhanden. Es läßt sich z. B. denken, daß die mangelhaften Zirkulationsverhältnisse im Gehirn hindernd auf die Funktionen desselben einwirken, oder wir können vermuten, daß die Aprosexie auf mehr indirektem Wege als eine durch die Nasenstenose ausgelöste nervöse Erscheinung entstanden ist.

Wenn wir nun auch ein gewisses Recht besitzen, die Ent- 
stehung der Aprosexie bei unsern Patienten in erster Linie der Nasenstenose zuzuschreiben, so sind wir doch keineswegs berechtigt, andere Kansalmomente answusehließen, unter anderem aus dem Grunde nicht, weil wir bei einer - wenn auch geringen - Anzahl Patienten keine Nasenstenose fanden. In vielen Fällen beruht sie unzweifelhaft auf einer weit mehr umfassenden Grundlage, so daß Schwerhörigkeit, Anämie, mangelhafte Ernäbrung und unvollkommene Körperentwicklung mit in das Spiel eintreten; hierfür spricht der Umstand, daß wir sie bei unsern Patienten hauptsächlich da fanden, wo die Symptome zahlreich und stark ausgesprochen vorhanden waren, wo mithin die Bedingungen eines weit tieferen Eingriffes in die Konstitution vorlagen, als die Nasenstenose allein hätte bewirken können.

Stottern und Stammeln. Unter den Patienten litten 18 an Stottern, 3 an Fehlern der Aussprache. Diese Zablen haben indes keinen Wert, wenn es sich um ein Urteil dartiber handelt, wie hänfig diese Komplikation im allgemeinen anftritt; denn die Stellung des Klinikvorstehers als Arzt des staatlichen Instituts für Sprachleidende wird natürlich dazu beitragen, daß eine unverhältnismäßig große Anzahl Sprachleidende die Klinik des Kommunebospitals aufsuchen.

Daß die adenoiden Vegetationen auch bezliglich der Pathogenese der Sprachmängel einen Platz behanpten, liegt außer allem Zweifel; es ist aber die Frage, wie großen Einfluß man ihnen beimessen soll. Möglicherweise liegt das Verhältnis so, daß der Sprachfehler als ein an und für sich rein nervöses Leiden von den Vegetationen in ungiunstiger Richtung beeinflußt wird, sodaß derselbe sich auf Grundlage der durch die adenoiden Vegetationen herbeigeftihrten pathologischen Zustände zu bedeutenden Stufen entwickelt. Von diesen Voraussetzungen ans erklärt es sich leicht, daß, den statistischen Aufklärungen zufolge, die adenoiden Vegetationen viel häufiger bei Stotterern auftreten, als bei normal redenden Individuen; denn man irrt wohl kaum in in der Anahme, daß es die höheren Stufen des Stotterns sind - mithin diejenigen Fälle, bei welchen man ein gleichzeitiges Vorbandensein von adenoiden Vegetationen vorauszusetzen berechtigt ist - welehe vorzugsweise zur Beobachtung kommen und demzufolge als statistisches Material in Anwendung gebracht werden. Daß selbst ein starkes Stottern ausnahmsweise im Anschluß an die Beseitigung der Vegetationen beilt, kann teils darauf beruhen, daß dureh die Adenotomie diejenige Ursache 
beseitigt wird, welehe den Sprachfehler auf seinem Höhepunkte erhält, teils aber auch auf der psychischen Einwirkung der Operation.

Der folgende aus unsern Journalen stammende Krankenbericht zeigt, daß anch auf einen Fehler der Aussprache durch die Adenotomie eingewirkt werden kann:

Rosa R., 9 Jahre alt, wurde der Klinik durch den Schularzt zugeführt, weil sie nicht imstande war, die Laute $k$. $g$ und $r$ auszusprecben. Sie sagt z. B. Josa statt Rosa, tann für kann. jeht für geht. Es finden sich eine leichte Nasenstenose und einige Neigung zu Angina, im ubrigen aber keine bervortretenden Symptome auf adenoide Vegetationen. Die objektive Untersuchung weist indes nach, daß außer einiger Hyperplasie der Pharynxtonsillen auch einige mittelgroße feste Vegetationen im Rhinopharynx vorhanden sind.

Adenotomie.

Nach der Operation bringt es die Patientin sebr scbnell dahin, die betreffenden Laute auszusprechen, und nach Ablauf einiger Monate ist ihre Sprache normal.

Aus dem obigen geht hervor, daß wir unter allen Umständen zur Ausführung der Adenotomie schreiten müssen, wenn adenoide Vegetationen in Verbindung mit einem Sprachfehler gefunden werden. Es läßt sich natürlich nicht im voraus sagen, in wie hohem Grade dabei auf den Sprachfehler eingewirkt werden wird; am häufigsten wird wohl die Einwirkung nicht besonders groß sein, und wir müssen daber zu einer methodisehen Behandlung des Sprachfehlers schreiten, haben aber alsdann gunstigere Bedingungen für die Erreichung eines befriedigenden Resultates herbeigeführt.

Mangelhafte geistige Entwicklung. Wenngleich die Aufmerksamkeit bei der Aufnahme der Journale auch auf etwaige Mängel der jntellektuellen Entwicklung der Patienten gerichtet war, so erscheinen die gesammelten Aufklärungen jedoch nicht als hinlänglich, wenn es sich um eine Entscheidung dessen handelt, wie hänfig solche Mängel gefunden worden sind. Der Einfluß der Vegetationen in der in Rede stehenden Beziehung liegt indes außer allem Zweifel. Wenn die Vegetationen sich im frühen Kindesalter einstellen, und wenn sie von Schwerhörigkeit und Störungen des physischen Entwicklungsprozesses begleitet werden, so kann man sich nur schwierig daneben eine normale Entwicklung der geistigen Vermögen vorstellen; deshalb finden wir denn auch unter den Patienten mit adenoiden Vegetationen eine nicht geringe Anzahl, welche in intellektueller Beziehung mangelhaft ausgertistet sind. Ist die geistige Entwicklungsstufe, auf welcher das Kind steht, niedrig, und hat dieser Zustand sehon lange gedauert, so dürfen wir nicht große Hoffnung auf eine durch die Adenotomie herbeizuführende Besserung des Zustandes setzen. 